\title{
Research Article \\ Finite Cartan Graphs Attached to Nichols Algebras of Diagonal Type
}

\author{
Chen Qian $(\mathbb{D})$ and Jing Wang \\ Department of Mathematics, School of Science, Beijing Forestry University, 100083 Beijing, China \\ Correspondence should be addressed to Jing Wang; wangjingmath@gmail.com
}

Received 15 May 2021; Accepted 12 July 2021; Published 19 August 2021

Academic Editor: Francesco Toppan

Copyright (C) 2021 Chen Qian and Jing Wang. This is an open access article distributed under the Creative Commons Attribution License, which permits unrestricted use, distribution, and reproduction in any medium, provided the original work is properly cited.

\begin{abstract}
Nichols algebras are fundamental objects in the construction of quantized enveloping algebras and in the classification of pointed Hopf algebras by the lifting method of Andruskiewitsch and Schneider. The structure of Cartan graphs can be attached to any Nichols algebras of diagonal type and plays an important role in the classification of Nichols algebras of diagonal type with a finite root system. In this paper, the main properties of all simply connected Cartan graphs attached to rank 6 Nichols algebras of diagonal type are determined. As an application, we obtain a subclass of rank 6 finite dimensional Nichols algebras of diagonal type.
\end{abstract}

\section{Introduction}

The theory of Nichols algebras is relatively young, but it has interesting applications to other research fields such as KacMoody Lie superalgebras [1] and conformal field theory [24]. Besides, it plays an important role in quantum groups [5-8].

The theory of Nichols algebras is motivated by the Hopf algebra theory. In any area of mathematics, the classification of all objects is very important. In Hopf algebra theory, the classification of all finite dimensional Hopf algebras is a tough question [5]. The structure of Nichols algebras appears naturally in the classification of pointed Hopf algebras in the following way. Given a Hopf algebra $H$, consider its coradical filtration

$$
H_{0} \subset H_{1} \subset \cdots,
$$

such that $H_{0}$ is a Hopf subalgebra of $H$ and the associated graded coalgebra

$$
\operatorname{gr} H=\underset{i}{\oplus} H_{i} / H_{i-1}
$$

Then, gr $H$ is a graded Hopf algebra, since the coradical $H_{0}$ of $H$ is a Hopf subalgebra. In addition, consider a projec- tion $\pi: \operatorname{gr} H \longrightarrow H_{0}$; let $R$ be the algebra of coinvariants of $\pi$. Then, by a result of Radford and Majid, $R$ is a braided Hopf algebra and grH is the bosonization (or biproduct) of $R$ and $H_{0}$ : gr $H \simeq R \# H_{0}$. The principle of the "lifting method" introduced in $[7,9]$ is first to study $R$, then to transfer the information to gr $H$ via bosonization, and finally to lift to $H$. The braided Hopf algebra $R$ is generated by the vector space $V$ of $H_{0}$-coinvariants of $H_{1} / H_{0}$, namely, Nichols algebra [9] $\mathscr{B}$ $(V)$ generated by $V$ in commemoration of W. Nichols [10] who started to study these objects as bialgebras of type one in 1978. Nichols algebras can be described in many different but alternative ways (see, for example, [11-15]).

The methods developed in the study of the generalizations of Lie algebras are also useful to analyze Nichols algebras [16]. Kharchenko proved that any Hopf algebra generated by skew-primitive and group-like elements has a restricted Poincaré-Birkhoff-Witt basis ([17], Theorem 2). Note that Kharchenko results can be applied to Nichols algebras of diagonal type. Motivated by the close relation to Lie theory, Heckenberger [18] defined the arithmetic root system and Weyl groupoid for Nichols algebras $\mathscr{B}(V)$ of diagonal type. Later, Cuntz and Heckenberger [19] and Heckenberger and Yamane [20] developed the combinatorial theory of these two structures. Then, the theory of root systems and 
Weyl groupoids was carried out in more general Nichols algebras [21-23]. Further, all finite Weyl groupoids were classified in $[24,25]$. Those constructions are very important theoretical tools for the classification of Nichols algebra $\mathscr{B}(V)$.

The crucial step to classify pointed Hopf algebras is to determine that all Nichols algebras $\mathscr{B}(V)$ is finite dimensional. Andruskiewitsch stated the following question.

Question 1 (Andruskiewitsch [5]). Given a braiding matrix $\left(q_{i j}\right)_{1 \leq i, j \leq \theta}$ whose entries are roots of 1 , when $\mathscr{B}(V)$ is finite-dimensional, where $V$ is a vector space with basis $x_{1}, \cdots, x_{\theta}$ and braiding $c\left(x_{i} \otimes x_{j}\right)=q_{i j}\left(x_{j} \otimes x_{i}\right)$ ? If so, compute $\operatorname{dim}_{\mathbb{k}} \mathscr{B}(V)$, and give a "nice" presentation by generators and relations.

Several authors obtained the classification result for infinite and finite dimensional Nichols algebra of Cartan type (see $[13,18,26])$. Heckenberger determined all finite dimensional Nichols algebra of diagonal type [27-30], that is, when $V$ is the direct sum of 1-dimensional Yetter-Drinfel'd modules over $H_{0}$. The generators and relations of such Nichols algebras were also given [31,32]. With the classification result, Andruskiewitsch and Schneider [33] obtained a classification theorem about finite-dimensional pointed Hopf algebras under some technical assumptions.

Based on such successful applications, the analysis to Nichols algebras over arbitrary fields is crucial and has also potential applications. Towards this direction, new examples of Nichols algebras in positive characteristic and a combinatorial formula to study the relations in Nichols algebras were found [34]. Over fields of positive characteristic, rank 2, rank 3 , and rank 4 finite dimensional Nichols algebras of diagonal type were listed in [35-37].

In this paper, we illustrate the main properties of the finite Cartan graphs of rank 6 attached to the Nichols algebras of diagonal type in Theorem 39. As a consequence, a subclass of rank 6 Nichols algebras of diagonal type with a finite root system is given in Section 5. The main algorithm in Section 3 is essential for our main goal.

Besides, the notations and conventions in [35-37] are followed, and several results from these papers will be used.

\section{Nichols Algebra of Diagonal Type}

In this section, we recall Yetter-Drinfel'd modules, braided vector spaces, and their relations. The main object of this paper is also presented. For further details on these topics, we refer to $[1,5,6]$.

2.1. Yetter-Drinfel'd Modules. Let $\mathbb{k}$ be a field of characteristic $p>0$. Let $\mathbb{k}^{*}=\mathbb{K} \backslash\{0\}, \mathbb{N}_{0}=\mathbb{N} \cup\{0\}, \theta \in \mathbb{N}_{0}$, and $I=\{1, \cdots$ $, \theta\}$. We start by recalling the main objects.

Definition 2. Let $V$ be a $\theta$-dimensional vector space over $\mathbb{k}$. The pair $(V, c)$ is called a braided vector space, if $c \in \operatorname{Aut}(V$ $\otimes V)$ is a solution of the braid equation, that is,

$$
(c \otimes \mathrm{id})(\mathrm{id} \otimes c)(c \otimes \mathrm{id})=(\mathrm{id} \otimes c)(c \otimes \mathrm{id})(\mathrm{id} \otimes c) .
$$

A braided vector space $(V, c)$ is termed of diagonal type if $V$ admits a basis $\left\{x_{i} \mid i \in I\right\}$ such that for all $i, j \in I$ one has

$$
c\left(x_{i} \otimes x_{j}\right)=q_{i j} x_{j} \otimes x_{i} \text { for some } q_{i j} \in \mathbb{K}^{*} .
$$

The matrix $\left(q_{i j}\right)_{i, j \in I}$ is termed of the braiding matrix of $V$. We say that the braiding matrix $\left(q_{i j}\right)_{i, j \in I}$ is indecomposable if for any $i \neq j$ there exists a sequence $i_{1}=i, i_{2}, \cdots, i_{t}=j$, of elements of $I$ such that $q_{i_{s} i_{s+1}} q_{i_{s+1} i_{s}} \neq 1$, where $1 \leq s \leq t-1$.

In this paper, we mainly concern the braided vector spaces of diagonal type with an indecomposable braiding matrix.

Definition 3. Let $H$ be a Hopf algebra. A Yetter-Drinfel'd module $V$ over $H$ is a left $H$-module with left action: $H \otimes V$ $\longrightarrow V$ and a left $H$-comodule with left coaction $\delta_{L}: V \longrightarrow$ $H \otimes V$ satisfying the compatibility condition

$$
\delta_{L}(h \cdot v)=h_{(1)} v_{(-1)} \kappa\left(h_{(3)}\right) \otimes h_{(2)} \cdot v_{(0)}, \quad h \in H, v \in V .
$$

We say that $V$ is of diagonal type if $H=\mathbb{k}^{\prime} G$ and $V$ is a direct sum of one-dimensional Yetter-Drinfel'd modules over the group algebra $\mathbb{k}^{\prime} G$, where $G$ is abelian.

We denote by ${ }_{H}^{H} \mathscr{Y} \mathscr{D}$ the category of Yetter-Drinfel'd modules over $H$, where morphisms preserve both the action and the coaction of $H$. The category ${ }_{H}^{H} y \mathscr{D}$ is braided with braiding

$$
c_{V, W}(v \otimes w)=v_{(-1)} \cdot w \otimes v_{(0)}
$$

for all $V, W \in_{H}^{H} \mathscr{Y} \mathscr{D}, v \in V$, and $w \in W$. Actually, the category ${ }_{H}^{H} y \mathscr{D}$ ia a braided monoidal category, where the monoidal structure is given by the tensor product over $\mathbb{k}$. Then, any Yetter-Drinfel'd module $V \epsilon_{H}^{H} \mathscr{Y} \mathscr{D}$ over $H$ admits a braiding $c_{V, V}$, and hence, $\left(V, c_{V, V}\right)$ is a braided vector space. Conversely, any braided vector space can be realized as a Yetter-Drinfel'd module over some Hopf algebras if and only if the braiding is rigid ([38], Section 2). Notice that YetterDrinfel'd module structures on $V$ with different Hopf algebras can give the same braiding and not all braidings of $V$ are induced by the above Equation (1).

If $H=\mathbb{k}^{\prime} G$, then we write ${ }_{G}^{G} \mathscr{Y} \mathscr{D}$ for the category of YetterDrinfel'd modules over $\mathbb{k}^{\prime} G$ and say that $V \in_{H}^{H} y \mathscr{D}$ is a Yetter-Drinfel'd module over $G$. Notice that if $V \in{ }_{G}^{G} \mathscr{Y} \mathscr{D}$ is of diagonal type, then $\left(V, c_{V, V}\right)$ is a braided vector space of diagonal type. Any braided vector space of diagonal type is also a Yetter-Drinfel'd module of diagonal type. Indeed, assume that $(V, c)$ is a braided vector space of diagonal type with an indecomposable braiding matrix $\left(q_{i j}\right)_{i, j \in I}$ of a basis $\left\{x_{i} \mid i \in I\right\}$. Let $G_{0}$ be a free abelian group generated by elements $\left\{g_{i} \mid i \in I\right\}$. Define the left coaction and left action by

$$
\delta_{L}\left(x_{i}\right)=g_{i} \otimes x_{i} \in G_{0} \otimes V, \quad g_{i} \cdot x_{j}=q_{i j} x_{j} \in V .
$$


Then, $V=\oplus_{i \in I} \mathbb{k} x_{i}$, and each $\mathbb{k} x_{i}$ is one-dimensional Yetter-Drinfel'd modules over $G_{0}$. Hence, $V$ is a YetterDrinfel'd module of diagonal type over $G_{0}$.

2.2. Nichols Algebras of Diagonal Type. Let $(V, c)$ be a $\theta$ -dimensional braided vector space of diagonal type. In this section, we recall a definition of the Nichols algebra $\mathscr{B}(V)$ generated by $(V, c)$. In order to do that, we introduce one more notion in the category ${ }_{H}^{H} y \mathscr{D}$.

Definition 4. Let $H$ be a Hopf algebra. A braided Hopf algebra in ${ }_{H}^{H} \mathscr{Y} \mathscr{D}$ is a 6-tuple $\mathscr{B}=\left(\mathscr{B}, \mu, 1, \delta, \varepsilon, \kappa_{B}\right)$, where $(B, \mu, 1)$ is an algebra in ${ }_{H}^{H} \mathscr{Y} \mathscr{D}$ and a coalgebra $(B, \delta, \varepsilon)$ in ${ }_{H}^{H} \mathscr{y} \mathscr{D}$, and $\kappa_{B}: B \longrightarrow B$ is a morphism in ${ }_{H}^{H} y \mathscr{D}$ such that $\delta$, $\varepsilon$, and $\kappa_{B}$ satisfy $\kappa_{B}\left(b^{(1)}\right) b^{(2)}=b^{(1)} \kappa_{B}\left(b^{(2)}\right)=\varepsilon(b) 1$, where we define $\delta(b)=b^{(1)} \otimes b^{(2)}$ as the coproduct of $\mathscr{B}$ to avoid the confusion.

Definition 5. The Nichols algebra generated by $V \epsilon_{H}^{H} \mathscr{Y} \mathscr{D}$ is defined as the quotient

$$
\mathscr{B}(V)=T(V) / \mathscr{I}(V)=\oplus_{n=0}^{\infty} V^{\otimes n} / \mathscr{I}(V),
$$

where $\mathscr{I}(V)$ is the unique maximal coideal among all coideals of $T(V)$ which are contained in $\oplus_{n \geq 2} V^{\otimes n}$. Nichols algebra $\mathscr{B}(V)$ is said to be of diagonal type if $V$ is a YetterDrinfel'd module of diagonal type. The dimension of $V$ is the rank of Nichols algebra $\mathscr{B}(V)$.

Note that if $B \in{ }_{H}^{H} y \mathscr{D}$ and $B$ is an algebra in ${ }_{H}^{H} y \mathscr{D}$, then $B \otimes B$ is an algebra in ${ }_{H}^{H} y \mathscr{D}$ with the product given by

$$
(a \otimes b)(c \otimes d)=a\left(b_{(-1)} \cdot c\right) \otimes b_{(0)} d,
$$

for all $a, b, c, d \in B$, where - denotes the left action of $H$ on $B$.

The tensor algebra $T(V)$ admits a natural structure of a Yetter-Drinfel'd module and an algebra structure in ${ }_{H}^{H} \mathscr{Y} \mathscr{D}$. It is then a braided Hopf algebra in ${ }_{H}^{H} y \mathscr{D}$ with coproduct $\delta$ $(v)=1 \otimes v+v \otimes 1 \in T(V) \otimes T(V)$ and counit $\varepsilon(v)=0$ for all $v \in V$ such that $\delta$ and $\varepsilon$ are the algebra morphisms. The antipode of $T(V)$ exists (see [7], Section 2.1). Notice that the product defined by Equation (9) on $T(V)$ is the unique algebra structure such that $\delta(v)=1 \otimes v+v \otimes 1 \in T(V) \otimes T(V)$ for all $v \in V$. The coproduct can be extended from $V$ to $T(V)$. For example, for all $v, w \in V$, one gets (we write the elements of $T(V)$ without the tensor product sign for brevity)

$$
\begin{aligned}
\delta(v w) & =\delta(v) \delta(w)=(1 \otimes v+v \otimes 1)(1 \otimes w+w \otimes 1) \\
& =1 \otimes v w+v_{(-1)} \cdot w \otimes v_{(0)}+v \otimes w+v w \otimes 1 .
\end{aligned}
$$

Let $\left(I_{i}\right)_{i \in I}$ be the family of all ideals of $T(V)$ contained in $\oplus_{n \geq 2} V^{\otimes n}$, i.e.,

$$
\delta\left(I_{i}\right) \subset I_{i} \otimes T(V)+T(V) \otimes I_{i} .
$$

Then, the coideal $\mathscr{I}(V):=\sum_{i \in I} I_{i}$ is the largest element in $\left(I_{i}\right)_{i \in I}$. Hence, $\mathscr{B}(V)$ is a braided Hopf algebra in ${ }_{H}^{H} \mathscr{Y} \mathscr{D}$. As proved in [6] (Proposition 3.2.12), Nichols algebra $\mathscr{B}(V)$ is the unique $\mathbb{N}_{0}$-graded braided Hopf algebra generated by $V$ in ${ }_{H}^{H} \mathscr{Y D}$ with homogenous components $\mathscr{B}(V)(0)=\mathbb{k}$, $\mathscr{B}(V)(1)=V$, and $P(\mathscr{B}(V))=V$, where $P(\mathscr{B}(V))$ is the space of primitive elements of $\mathscr{B}(V)$.

2.3. Weyl Groupoids. In this section, we recall the notations of semi-Cartan graphs, root systems, and Weyl groupoids. We mainly follow the terminology from [20,39] (see also [35-37]).

Definition 6. A generalized Cartan matrix is a matrix $A=\left(a_{i j}\right)_{i, j \in I}$ with integer entries such that

(i) $a_{i i}=2$ and $a_{j k} \leq 0$ for any $i, j, k \in I$ with $j \neq k$

(ii) if $a_{i j}=0$ for some $i, j \in I$, then $a_{j i}=0$

A generalized Cartan matrix $A \in \mathbb{Z}^{I \times I}$ is decomposable if there exists a nonempty proper subset $I_{1} \subset I$ such that $a_{i j}=$ 0 for any $i \in I_{1}$ and $j \in I \backslash I_{1}$. We say that $A$ is indecomposable if $A$ is not decomposable.

Definition 7. Let $\mathscr{X}$ be a nonempty set and $A^{X}=\left(a_{i j}^{X}\right)_{i, j \in I}$ a generalized Cartan matrix for all $X \in \mathscr{X}$. For any $i \in I$, let $r_{i}: \mathscr{X} \longrightarrow \mathscr{X}, X \mapsto r(i, X)$, where $r: I \times \mathscr{X} \longrightarrow \mathscr{X}$ is a map. The quadruple

$$
\mathscr{C}=\mathscr{C}\left(I, \mathscr{X}, r,\left(A^{X}\right)_{X \in \mathscr{X}}\right)
$$

is called a semi-Cartan graph if $r_{i}^{2}=\mathrm{id}_{\mathscr{X}}$ for all $i \in I$, and $a_{i j}^{X}$ $=a_{i j}^{r_{i}(X)}$ for all $X \in \mathscr{X}$ and $i, j \in I$. We say that a semi-Cartan graph $\mathscr{C}$ is indecomposable if $A^{X}$ is indecomposable for all $X \in \mathscr{X}$.

For the sake of simplicity, the elements of the set $\left\{r_{i}(X), i \in I\right\}$ are termed the neighbors of $X$ for all $X \in \mathscr{X}$. The cardinality of $I$ is termed the rank of $\mathscr{C}$, and the elements of $\mathscr{X}$ are the points of $\mathscr{C}$.

Definition 8. A semi-Cartan graph $\mathscr{C}$ is standard, if $A^{X}=A^{Y}$ for all $X, Y \in O b(\mathscr{W}(\mathscr{C}))$.

Definition 9. The exchange graph of $\mathscr{C}$ is a labeled nonoriented graph with vertices set $\mathscr{X}$ and edges set $I$, where two vertices $X, Y$ are connected by an edge $i$ if and only if $X \neq Y$ and $r_{i}(X)=Y$ (and $r_{i}(Y)=X$ ). We display one edge with several labels instead of several edges for simplification. A semiCartan graph $\mathscr{C}$ is said to be connected if its exchange graph is connected.

For the remaining part of this section, we assume that $\mathscr{C}=\mathscr{C}\left(I, \mathscr{X}, r,\left(A^{X}\right)_{X \in \mathscr{X}}\right)$ is a connected semi-Cartan graph. Let $\left(\alpha_{i}\right)_{i \in I}$ be the standard basis of $\mathbb{Z}^{I}$. For all $X \in \mathscr{X}$, let 


$$
s_{i}^{X} \in \operatorname{Aut}\left(\mathbb{Z}^{I}\right), \quad s_{i}^{X} \alpha_{j}=\alpha_{j}-a_{i j}^{X} \alpha_{i},
$$

for all $j \in I$. Let $\mathscr{D}(\mathscr{X}, I)$ be the category such that $\operatorname{Ob} \mathscr{D}(\mathscr{X}, I)=\mathscr{X}$ and morphisms $\operatorname{Hom}(X, Y)=\{(Y, f, X) \mid f$ $\left.\in \operatorname{End}\left(\mathbb{Z}^{I}\right)\right\}$ for $X, Y \in \mathscr{X}$ with the composition $(Z, g, Y) \circ($ $Y, f, X)=(Z, g f, X)$ for all $X, Y, Z \in \mathscr{X}, f, g \in \operatorname{End}\left(\mathbb{Z}^{I}\right)$. Let $\mathscr{W}(\mathscr{C})$ be the smallest subcategory of $\mathscr{D}(\mathscr{X}, I)$, where the morphisms are generated by $\left(r_{i}(X), s_{i}^{X}, X\right)$, with $i \in I, X \in \mathscr{X}$ . From now on, we write $s_{i}^{X}$ instead of $\left(r_{i}(X), s_{i}^{X}, X\right)$, if no confusion is possible. Notice that all generators $s_{i}^{X}$ are reflections and hence are invertible. Then, $\mathscr{W}(\mathscr{C})$ is a groupoid.

For any category $\mathscr{D}$ and any object $X$ in $\mathscr{D}$, let $\operatorname{Hom}(\mathscr{D}$, $X)=\cup_{Y \in \mathscr{D}} \operatorname{Hom}(Y, X)$.

Definition 10. For all $X \in \mathscr{X}$, the set

$$
\Delta^{X \mathrm{re}}=\left\{\omega \alpha_{i} \in \mathbb{Z}^{I} \mid \omega \in \operatorname{Hom}(\mathscr{W}(\mathscr{C}), X)\right\}
$$

is called the set of real roots of $\mathscr{C}$ at $X$. The elements of $\Delta_{+}^{X \text { re }}=\Delta^{X \text { re }} \cap \mathbb{N}_{0}^{I}$ are called positive roots and those of $\Delta^{X \text { re }}$ $\cap-\mathbb{N}_{0}^{I}$ negative roots, denoted by $\Delta_{-}^{X}$ re. If the set $\Delta^{X \text { re }}$ is finite for all $X \in \mathscr{X}$, then we say that $\mathscr{C}$ is finite.

Definition 11 . We say that $\mathscr{R}=\mathscr{R}\left(\mathscr{C},\left(\Delta^{X}\right)_{X \in \mathscr{X}}\right)$ is a root system of type $\mathscr{C}$ if for all $X \in \mathscr{X}$, the sets $\Delta^{X}$ are the subsets of $\mathbb{Z}^{I}$ such that

$$
\Delta^{X}=\left(\Delta^{X} \cap \mathbb{N}_{0}^{I}\right) \cup-\left(\Delta^{X} \cap \mathbb{N}_{0}^{I}\right)
$$

(i) $\Delta^{X} \cap \mathbb{Z} \alpha_{i}=\left\{\alpha_{i},-\alpha_{i}\right\}$ for all $i \in I$

(ii) $s_{i}^{X}\left(\Delta^{X}\right)=\Delta^{r_{i}(X)}$ for all $i \in I$

(iii) $\left(r_{i} r_{j}\right)^{m_{i j}^{X}}(X)=X$ for any $i, j \in I$ with $i \neq j$ where $m_{i j}^{X}$ $=\left|\Delta^{X} \cap\left(\mathbb{N}_{0} \alpha_{i}+\mathbb{N}_{0} \alpha_{j}\right)\right|$ is finite

We say that $\mathscr{W}(R):=\mathscr{W}(\mathscr{C})$ is the groupoid of $\mathscr{R}$. As in [13] (Definition 4.3), we say that $\mathscr{R}$ is reducible if there exist nonempty disjoint subsets of $I^{\prime}, I^{\prime \prime} \subset I$ such that $I=I^{\prime} \cup I^{\prime \prime}$ and $a_{i j}=0$ for all $i \in I^{\prime}, j \in I^{\prime \prime}$ and

$$
\Delta^{X}=\left(\Delta^{X} \cap \sum_{i \in I^{\prime}} \mathbb{Z} \alpha_{i}\right) \cup\left(\Delta^{X} \cap \sum_{j \in I^{\prime \prime}} \mathbb{Z} \alpha_{j}\right) \text { for all } X \in \mathscr{X}
$$

In this case, we write $\mathscr{R}=\left.\left.\mathscr{R}\right|_{I_{1}} \oplus \mathscr{R}\right|_{I_{2}}$. If $\mathscr{R} \neq\left.\left.\mathscr{R}\right|_{I_{1}} \oplus \mathscr{R}\right|_{I_{2}}$ for all nonempty disjoint subsets $I_{1}, I_{2} \subset I$, then $\mathscr{R}$ is termed irreducible.

Definition 12. Let $\mathscr{R}=\mathscr{R}\left(\mathscr{C},\left(\Delta^{X}\right)_{X \in \mathscr{X}}\right)$ be a root system of type $\mathscr{C}$. We say that $\mathscr{R}$ is finite if $\Delta^{X}$ is finite for all $X \in \mathscr{X}$.
Let $\mathscr{R}=\mathscr{R}\left(\mathscr{C},\left(\Delta^{X}\right)_{X \in \mathscr{X}}\right)$ be a root system of type $\mathscr{C}$. We recall some properties of $\mathscr{R}$ from $[19,24]$.

Lemma 13. Let $X \in \mathscr{X}, k \in \mathbb{Z}$, and $i, j \in I$ such that $i \neq j$. Then $\alpha_{j}+k \alpha_{i} \in \Delta^{X \text { re }}$ if and only if $0 \leq k \leq-a_{i j}^{X}$.

Notice that the finiteness of $\mathscr{R}$ does not mean that $\mathscr{W}(\mathscr{R})$ is also finite, since the set $\mathscr{X}$ might be infinite.

Lemma 14. Let $\mathscr{C}=\mathscr{C}\left(I, \mathscr{X}, r,\left(A^{X}\right)_{X \in \mathscr{X}}\right)$ be a connected semiCartan graph and $\mathscr{R}=\mathscr{R}\left(\mathscr{C},\left(\Delta^{X}\right)_{X \in \mathscr{X}}\right)$ be a root system of type $\mathscr{C}$. Then, the following are equivalent.

(1) $\mathscr{R}$ is finite

(2) $\Delta^{X}$ is finite for some $X \in \mathscr{X}$

(3) $\mathscr{C}$ is finite

(4) $\mathscr{W}(\mathscr{R})$ is finite

Recall that $\mathscr{C}$ is a connected semi-Cartan graph. Then, we get the following.

Proposition 15. Let $\mathscr{R}=\mathscr{R}\left(\mathscr{C},\left(\Delta^{X}\right)_{X \in \mathscr{X}}\right)$ be a root system of type $\mathscr{C}$. Then, the following are equivalent.

(1) There exists $X \in \mathscr{X}$ such that $A^{X}$ is indecomposable

(2) The semi-Cartan graph $\mathscr{C}$ is indecomposable

If $\mathscr{R}$ is finite, then the semi-Cartan graph $\mathscr{C}$ is indecomposable if and only if the root system $\mathscr{R}$ is irreducible.

Definition 16. We say $\mathscr{C}$ is a Cartan graph if the following hold:

(i) For all $X \in \mathscr{X}$, the set $\Delta^{X \text { re }}=\Delta_{+}^{X \text { re }} \cup \Delta_{-}^{X \text { re }}$

(ii) If $l_{m n}^{Y}:=\left|\Delta^{Y \text { re }} \cap\left(\mathbb{N}_{0} \alpha_{m}+\mathbb{N}_{0} \alpha_{n}\right)\right|$ is finite, then $\left(r_{m} r_{n}\right)^{l_{m n}^{Y}}(Y)=Y$, where $m, n \in I, Y \in \mathscr{X}$

In this case, $\mathscr{W}(\mathscr{C})$ is called the Weyl groupoid of $\mathscr{C}$.

Let $\mathscr{R}^{\text {re }}:=\mathscr{R}\left(\mathscr{C},\left(\Delta^{X \text { re }}\right)_{X \in \mathscr{X}}\right)$. Then, $\mathscr{C}$ is a Cartan graph if and only if $\mathscr{R}^{\text {re }}$ is a root system of type $\mathscr{C}$. Indeed, we get that $s_{i}^{X}\left(\Delta^{X \text { re }}\right)=\Delta^{r_{i}(X) \text { re }}$ by Equation (14). For all $X \in \mathcal{X}$, we obtain that $\Delta^{X \text { re }}=\Delta_{+}^{X \text { re }} \cup \Delta_{-}^{X \text { re }}$, since $\omega s_{i}^{r_{i}(X)}\left(\alpha_{i}\right)=-\omega\left(\alpha_{i}\right)$ for any $\omega$ $\in \operatorname{Hom}(\mathscr{W}(\mathscr{C}), X)$.

The following proposition implies that if $\mathscr{R}$ is a finite root system of type $\mathscr{C}$, then $\mathscr{R}=\mathscr{R}^{\text {re }}$; namely, all roots are real and $\mathscr{R}$ is uniquely determined by $\mathscr{C}$.

Proposition 17. Let $\mathscr{R}=\mathscr{R}\left(\mathscr{C},\left(\Delta^{X}\right)_{X \in \mathscr{X}}\right)$ be a root system of type $\mathscr{C}$. Let $X \in \mathscr{X}, m \in \mathbb{N}_{0}$, and $i_{1}, \cdots, i_{m} \in I$ such that

$$
\omega=i d_{X} s_{i_{1}} s_{i_{2}} \cdots s_{i_{m}} \in \operatorname{Hom}(\mathscr{W}(\mathscr{C}), X)
$$


and $\ell(\omega)=m$. Then, the elements

$$
\beta_{n}=i d_{X} s_{i_{1}} s_{i_{2}} \cdots s_{i_{n-1}}\left(\alpha_{i_{n}}\right) \in \Delta^{X} \cap \mathbb{N}_{0}^{I}
$$

are pairwise different, where $n \in\{1,2, \cdots, m\}$ (and $\beta_{1}=\alpha_{i_{1}}$ ). Here,

$$
\ell(\omega)=\min \left\{m \in \mathbb{N}_{0} \mid \omega=i d_{X} s_{i_{1}} s_{i_{2}} \cdots s_{i_{m}}, i_{1}, i_{2}, \cdots, i_{m} \in I\right\}
$$

is the length of $\omega \in \operatorname{Hom}(\mathscr{W}(\mathscr{C}), X)$. In particular, if $\mathscr{R}$ is finite and $\omega \in \operatorname{Hom}(\mathscr{W}(\mathscr{C}))$ is the longest element, then

$$
\left\{\beta_{n} \mid 1 \leq n \leq \ell(\omega)=\frac{\left|\Delta^{X}\right|}{2}\right\}=\Delta^{X} \cap \mathbb{N}_{0}^{I} .
$$

Remark 18. If $\mathscr{C}$ is a finite Cartan graph, then $\mathscr{R}$ is finite, and hence,

$$
\mathscr{R}^{\mathrm{re}}=\mathscr{R}^{\mathrm{re}}\left(\mathscr{C},\left(\Delta^{\mathrm{X} \mathrm{re}}\right)_{X \in \mathscr{X}}\right)
$$

is the unique root system of type $\mathscr{C}$ by Proposition 17; that is, $\mathscr{R}$ is uniquely determined by $\mathscr{C}$.

2.4. Cartan Graphs for Nichols Algebras of Diagonal Type. In this section, we attach a semi-Cartan graph to a tuple of finite-dimensional Yetter-Drinfel'd modules under some finiteness conditions. Let $G$ be an abelian group. Let $\mathscr{F}_{\theta}^{G}$ be the set of $\theta$-tuples of finite-dimensional irreducible objects in ${ }_{G}^{G} \mathcal{Y} \mathscr{D}$ and $\mathscr{X}_{\theta}^{G}$ be the set of $\theta$-tuples of isomorphism classes of finite-dimensional irreducible objects in ${ }_{G}^{G} \mathscr{Y} \mathscr{D}$. For any $\left(M_{1}, \cdots, M_{\theta}\right) \in \mathscr{F}_{\theta}^{G}$, write $[M]:=\left(\left[M_{1}\right], \cdots,\left[M_{\theta}\right]\right) \in \mathscr{X}_{\theta}^{G}$ the corresponding isomorphism class of $\left(M_{1}, \cdots, M_{\theta}\right)$.

Assume that $V_{M}=\oplus_{i \in I} \mathbb{k} x_{i} \in_{G}^{G} \mathscr{Y} \mathscr{D}$ is a Yetter-Drinfel'd module of diagonal type over $G$, where $\left\{x_{i} \mid i \in I\right\}$ is a basis of $V$. Then, there exists a matrix $\left(q_{i j}\right)_{i, j \in I}$ such that $\delta\left(x_{i}\right)=$ $g_{i} \otimes x_{i}$ and $g_{i} \cdot x_{j}=q_{i j} x_{j}$ for all $i, j \in I$. We fix that $M=\left(\mathbb{k} x_{1}\right.$, $\left.\mathbb{R}_{k} x_{2}, \cdots, \mathbb{k}_{k} x_{\theta}\right) \in \mathscr{F}_{\theta}^{G}$ is a tuple of one-dimensional YetterDrinfel'd over $G$ and $[M] \in \mathscr{X}_{\theta}^{G}$. We say that the matrix $\left(q_{i j}\right)_{i, j \in I}$ is the braiding matrix of $M$. Recall that the matrix is independent of the basis $\left\{x_{i} \mid i \in I\right\}$ up to permutation of I. We say $\mathscr{B}\left(V_{M}\right)=\mathscr{B}\left(\oplus_{i=1}^{n} \mathbb{k} x_{i}\right)$ is the Nichols algebra of the tuple $M$, denoted by $\mathscr{B}(M)$.

Recall that the adjoint representation ad of a Nichols algebra $\mathscr{B}(V)$ from [9] is the linear map $\operatorname{ad}_{c}: V \longrightarrow$ End $(\mathscr{B}(V))$

$$
\operatorname{ad}_{c} x(y)=\mu(\mathrm{id}-c)(x \otimes y)=x y-\left(x_{(-1)} \cdot y\right) x_{(0)}
$$

for all $x \in V, y \in \mathscr{B}(V)$, where $\mu$ is the multiplication map of $\mathscr{B}(V)$ and $c$ is defined by Equation (6). In particular, the braided commutator $\mathrm{ad}_{c}$ of $\mathscr{B}(M)$ takes the form

$$
\operatorname{ad}_{c} x_{i}(y)=x_{i} y-\left(g_{i} \cdot y\right) x_{i} \text { for all } i \in I, y \in \mathscr{B}(M) \text {. }
$$

In order to construct a semi-Cartan graph to $M$, we recall some finiteness conditions from [7, 22].

Definition 19. Let $i \in I$. We say that $M$ is $i$-finite, if for any $j \in I \backslash\{i\},\left(\operatorname{ad}_{c} x_{i}\right)^{m}\left(x_{j}\right)=0$ for some $m \in \mathbb{N}$.

Lemma 20. For any $i, j \in I$ with $i \neq j, s$, the following are equivalent.

(i) $(m+1)_{q_{i i}}\left(q_{i i}^{m} q_{i j} q_{j i}-1\right)=0$ and $(k+1)_{q_{i i}}\left(q_{i i}^{k} q_{i j} q_{j i}-1\right)$ $\neq 0$ for all $0 \leq k<m$

(ii) $\left(a d_{c} x_{i}\right)^{m+1}\left(x_{j}\right)=0$ and $\left(a d_{c} x_{i}\right)^{m}\left(x_{j}\right) \neq 0$ in $\mathscr{B}(V)$

Here, $(n)_{q}:=1+q+\cdots+q^{n-1}$, which is 0 if and only if $q^{n}=1$ for $q \neq 1$ or $p \mid n$ for $q=1$. Notice that $(1)_{q} \neq 0$ for any $q \in \mathbb{N}$.

Hence, we get the following from Lemma 20.

Lemma 21. Let $i \in I$. Then, $M=\left(\mathbb{k} x_{j}\right)_{j \in I}$ is $i$-finite if and only if for any $j \in I \backslash\{i\}$ there is a nonnegative integer $m$ satisfying $(m+1) q_{i i}\left(q_{i i}^{m} q_{i j} q_{j i}-1\right)=0$.

Let $i \in I$. Assume that $M$ is $i$-finite. Let $\left(a_{i j}^{M}\right)_{j \in I} \in \mathbb{Z}^{I}$ and $R_{i}(M)=\left(R_{i}(M)_{j}\right)_{j \in I}$, where

$$
\begin{gathered}
a_{i j}^{M}=\left(\begin{array}{ll}
2, & \text { if } j=i, \\
-\max \left\{m \in \mathbb{N}_{0} \mid\left(\operatorname{ad}_{c} x_{i}\right)^{m}\left(x_{j}\right) \neq 0\right\}, & \text { if } j \neq i,
\end{array}\right. \\
R_{i}(M)_{i}=\mathbb{k} y_{i}, R_{i}(M)_{j}=\mathbb{k}\left(\operatorname{ad}_{c} x_{i}\right)^{-a_{i j}^{M}}\left(x_{j}\right),
\end{gathered}
$$

where $y_{i} \in\left(\mathbb{k} x_{i}\right)^{*} \backslash\{0\}$. If $M$ is not $i$-finite, then let $R_{i}(M)=M$. Then, $R_{i}(M)$ is a $\theta$-tuple of one-dimensional Yetter-Drinfel'd modules over $G$.

Let

$$
\begin{aligned}
& \mathscr{F}_{\theta}^{G}(M)=\left\{R_{i_{1}} \cdots R_{i_{n}}(M) \in \mathscr{F}_{\theta}^{G} \mid n \in \mathbb{N}_{0}, i_{1}, \cdots, i_{n} \in I\right\} \\
& \mathscr{X}_{\theta}^{G}(M)=\left\{\left[R_{i_{1}} \cdots R_{i_{n}}(M)\right] \in \mathscr{X}_{\theta}^{G} \mid n \in \mathbb{N}_{0}, i_{1}, \cdots, i_{n} \in I\right\} .
\end{aligned}
$$

Definition 22. We say that $M$ admits all reflections if $N$ is $i$ -finite for all $N \in \mathscr{F}_{\theta}^{G}(M)$.

Notice that the reflections depend only on the braiding matrix $\left(q_{i j}\right)_{i, j \in I}$. We recall the notion of generalized Dynkin diagram for a braided vector space of diagonal type [40].

Definition 23. Let $V$ be a $\theta$-dimensional braided vector space of diagonal type with the braiding matrix $\left(q_{i j}\right)_{i, j \in I}$. The generalized Dynkin diagram of $V$ is a nondirected graph $\mathscr{D}$ with the following properties: 
(i) There is a bijective map $\phi$ from $I$ to the vertices of $\mathscr{D}$

(ii) For all $i \in I$, the vertex $\phi(i)$ is labeled by $q_{i i}$

(iii) For all $i, j \in I$ with $i \neq j$, the number $n_{i j}$ of edges between $\phi(i)$ and $\phi(j)$ is either 0 or 1 . If $q_{i j} q_{j i}=1$, then $n_{i j}=0$; otherwise, $n_{i j}=1$ and the edge is labeled by $q_{i j} q_{j i}$

We say that the generalized Dynkin diagram of $M$ is the generalized Dynkin diagram of braided vector space $\oplus_{i \in I}$ $M_{i}$. Notice that the generalized Dynkin diagram of $M$ is connected if the braiding matrix of $M$ is indecomposable.

In more details, one can obtain the labels of the generalized Dynkin diagram of $R_{i}(M)=\left(R_{i}(M)_{j}\right)_{j \in I}$ by the following lemma.

Lemma 24. Let $i \in I$. Assume that $M$ is $i$-finite and let $a_{i j}:=a_{i j}^{M}$ for all $j \in I$. Let $\left(q^{\prime}{ }_{j k}\right)_{j, k \in I}$ be the braiding matrix of $R_{i}(M)$ with respect to $\left(y_{j}\right)_{j \in I}$. Then,

$$
\begin{aligned}
& q_{j j}{ }^{\prime}=\left(\begin{array}{ll}
q_{i i}, & \text { if } j=i, \\
q_{j j}, & \text { if } j \neq i, q_{i j} q_{j i}=q_{i i}^{a_{i j}}, \\
q_{i i} q_{j j}\left(q_{i j} q_{j i}\right)^{-a_{i j}}, & \text { if } j \neq i, q_{i i} \in G^{\prime}{ }_{1-a_{i j}}, \\
q_{j j}\left(q_{i j} q_{j i}\right)^{-a_{i j}}, & \text { if } j \neq i, q_{i i}=1,
\end{array}\right. \\
& q_{i j}{ }^{\prime} q_{j i}{ }^{\prime}=\left(\begin{array}{ll}
q_{i j} q_{j i}, & \text { if } j \neq i, q_{i j} q_{j i}=q_{i i}^{a_{i j}}, \\
q_{i i}^{2}\left(q_{i j} q_{j i}\right)^{-1}, & \text { if } j \neq i, q_{i i} \in G^{\prime}{ }_{1-a_{i j}}, \\
\left(q_{i j} q_{j i}\right)^{-1}, & \text { if } j \neq i, q_{i i}=1,
\end{array}\right. \\
& q_{j k}{ }^{\prime} q_{k j}{ }^{\prime}=\left(\begin{array}{ll}
q_{j k} q_{k j}, & \text { if } q_{i r} q_{r i}=q_{i i}^{a_{i r}}, r \in\{j, k\}, \\
q_{j k} q_{k j}\left(q_{i k} q_{k i} q_{i i}^{-1}\right)^{-a_{i j}}, & \text { if } q_{i j} q_{j i}=q_{i i}^{a_{i j}}, q_{i i} \in G^{\prime}{ }_{1-a_{i k}}, \\
q_{j k} q_{k j}\left(q_{i j} q_{j i}\right)^{-a_{i k}}\left(q_{i k} q_{k i}\right)^{-a_{i j},}, & \text { if } q_{i i}=1, \\
q_{j k} q_{k j} q_{i i}^{2}\left(q_{i j} q_{j i} q_{i k} q_{k i}\right)^{-a_{i j}}, & \text { if } q_{i i} \in G^{\prime}{ }_{1-a_{i k}}, q_{i i} \in G^{\prime}{ }_{1-a_{i j}},
\end{array}\right.
\end{aligned}
$$

for $j \neq k,|j-i|=1,|k-i|=1$. For $|j-i|>1,|k-i|>1$, $q_{j k}^{\prime} q_{k j}^{\prime}=q_{j k} q_{k j}$. Here, $G_{n^{\prime}}$ denotes the set of primitive $n$th roots of unity in $\mathbb{k}$, that is, $G_{n}^{\prime}=\left\{q \in \mathbb{k}^{*} \mid q^{n}=1, q^{k} \neq 1\right.$ for all $1 \leq k$ $<n\}$ for $n \in \mathbb{N}$.

If $M$ admits all reflections, then we are able to construct a semi-Cartan graph $\mathscr{C}(M)$ of $M$ by ([36], Proposition 1.5).

Theorem 25. Assume that $M$ admits all reflections. For all $X \in \mathscr{X}_{\theta}^{G}(M)$, let $[X]_{\theta}=\left\{Y \in \mathscr{X}_{\theta}^{G}(M) \mid Y\right.$ and $X$ have the same generalized Dynkin diagram $\}$.
Let $\mathscr{Y}_{\theta}(M)=\left\{[X]_{\theta} \mid X \in \mathscr{X}_{\theta}^{G}(M)\right\}$ and $A^{[X]_{\theta}}=A^{X}$ for all $X \in \mathscr{X}_{\theta}^{G}(M)$. Let $t: I \times \mathscr{Y}_{\theta}(M) \longrightarrow \mathscr{Y}_{\theta}(M), \quad\left(i,[X]_{\theta}\right) \mapsto$ $\left[R_{i}(X)\right]_{\theta}$. Then, the tuple

$$
\mathscr{C}(M)=\left\{I, \mathscr{Y}_{\theta}(M), t,\left(A^{Y}\right)_{Y \in \mathscr{Y}_{\theta}(M)}\right\}
$$

is a connected semi-Cartan graph. We say that $\mathscr{C}(M)$ is the semi-Cartan graph attached to $M$.

Furthermore, one can attach a groupoid $\mathscr{W}(M):=\mathscr{W}(\mathscr{C}$ $(M))$ to $M$ if $M$ admits all reflections.

Notice that Nichols algebra $\mathscr{B}(M)$ is $\mathbb{N}_{0}^{\theta}$-graded with $\operatorname{deg} M_{i}=\alpha_{i}$ for all $i \in I$. Following the terminology in [22], we say that the Nichols algebra $\mathscr{B}(M)$ is decomposable if there exists a totally ordered index set $(L, \leq)$ and a sequence $\left(W_{l}\right)_{l \in L}$ of finite-dimensional irreducible $\mathbb{N}_{0}^{\theta}$-graded objects in ${ }_{G}^{G} \mathscr{Y} \mathscr{D}$ such that

$$
\mathscr{B}(M) \simeq \underset{l \in L}{\otimes} \mathscr{B}\left(W_{l}\right)
$$

For each decomposition (28), we define the set of positive roots $\Delta_{+}^{[M]} \subset \mathbb{Z}^{I}$ and the set of roots $\Delta^{[M]} \subset \mathbb{Z}^{I}$ of $[M]$ by

$$
\Delta_{+}^{[M]}=\left\{\operatorname{deg}\left(W_{l}\right) \mid l \in L\right\}, \Delta^{[M]}=\Delta_{+}^{[M]} \cup-\Delta_{+}^{[M]} .
$$

By [22] (Theorem 4.5), we obtain that the set of roots $\Delta^{[M]}$ of $[M]$ does not depend on the choice of the decomposition.

Remark 26. If $\operatorname{dim} M_{i}=1$ for all $i \in I$, then the Nichols algebra $\mathscr{B}(M)$ is decomposable based on the theorem of Kharchenko ([17], Theorem 2). The set of roots of Nichols algebra $\mathscr{B}(M)$ can be always defined, and it is denoted by $\Delta^{[M]}$. If the set of roots $\Delta^{[M]}$ is finite, then we can check that $M$ admits all reflections by [22] (Corollary 6.12).

If $M$ admits all reflections and $\Delta^{[M]}$ is finite, then we can define a finite root system $\mathscr{R}(M)\left(\mathscr{C}(M),\left(\Delta^{[N]}\right)_{N \in \mathscr{F}_{\theta}^{G}(M)}\right)$ of type $\mathscr{C}(M)$.

Theorem 27. Assume that $M$ admits all reflections. Then, the following are equivalent.

(1) $\Delta^{[M]}$ is finite

(2) $\mathscr{C}(M)$ is a finite Cartan graph

(3) $\mathscr{W}(M)$ is finite

(4) $\mathscr{R}(M):=\mathscr{R}(M)\left(\mathscr{C}(M),\left(\Delta^{[N]}\right)_{N \in \mathscr{F}_{\theta}^{G}(M)}\right)$ is finite

In all cases, $\mathscr{R}(M)$ is the unique root system of type $\mathscr{C}(M)$.

Proof. Since $M$ is a $\theta$-tuple of one-dimensional YetterDrinfel'd modules, we obtain that the Nichols algebra $\mathscr{B}(M)$ generated by $V_{M}$ is decomposable, and hence, $\Delta^{[M]}$ is defined. Then, $\mathscr{R}(M)=\mathscr{R}(M)\left(\mathscr{C}(M),\left(\Delta^{[N]}\right)_{N \in \mathscr{F}_{\theta}^{G}(M)}\right)$ is 
Check the neighborhoods of root systems which have Cartan matrix of type $A_{6}$.

Input: all root systems in Appendix 5.

Output: All the $A_{6}$ neighborhoods

1. Copy the data in Appendix 5 to set $r$ and allrs.

2. Call Sort $(r s$, cmpvect $)$.

3. Call Reflection $(\boldsymbol{r s}, \boldsymbol{i})$, for $i \in\{1, \cdots, 6\}$ and sort all the reflected root systems. Check if the reflections yield new root systems. If yes, then sort them and add to the set allrs. Repeat above to all new found root systems and update the set allrs until the reflections yield no new root systems.

4. Call $\mathbf{c m}(\operatorname{allrs}[i])$, for $i=1, \cdots$, Length(allrs). Check if its Cartan matrix is of type $A_{6}$ by calling IsTypeA6(cm(allrs[i])). Collect root systems which have Cartan matrix of finite type of A6 to set "A6roots".

5. Reflect all rank 6 root systems in set "A6roots" in 6 directions and remember all the positions of the reflected root systems in allrs.

6. Return part of the Exchange graph including all objects with Cartan matrix of type $A_{6}$ and the Cartan matrixes of the reflected root systems.

Algorithm 28: GoodNeighborhoods $(r s)$.

the root system of type $\mathscr{C}(M)$ by [22] (Theorem 6.11). Hence, the claim is true by Lemma 14.

\section{Calculation of Finite Cartan Graphs}

In this section, we obtain the properties of finite connected indecomposable Cartan graphs attached to Nichols algebras of diagonal type with a finite root system and present the results in Theorem 39.

Our algorithms described below are sufficiently powerful in finding the properties, which are extremely essential for our classification. The implementation in Shell terminates within a few seconds on a usual computer.

3.1. The Idea. For convenience, we copy the irreducible root systems of rank 6 in [25] (Appendix B) to Appendix 5.

This is an overview of the main algorithm without any details.

\subsection{Some Remarks}

Remark 29. Before starting the algorithms, we give several remarks on Algorithm 28.

(1) In step 1, we restrict the input root systems to Appendix 5. In details, since Cuntz and Heckenberger illustrate all the finite Weyl groupoids in [25], the finite root system of Nichols algebras of diagonal type must be contained in [25] (Cor. 3.13 Appendix B). The classification of Nichols algebras of diagonal type with a finite root system which appears in [25] (Cor. 3.13) is not difficult to achieve

(2) From steps 1 to 3, we obtain all the possible reflected root systems of the given root system rs. By Lemma 14, the Weyl groupoid is finite. Hence, in step 3, the implementation of the reflections terminates

(3) From Table 1 of Appendix A in [25], for each root system $r s$, there is an object such that the generalized Cartan matrix is of type $A_{6}$. Hence, for simplification,
TABLE 1: Finite dimensional Nichols algebra of diagonal type over $\mathbb{k}$ $(p \neq 3)$.

\begin{tabular}{|c|c|c|}
\hline$X$ & Gener. Dynkin diagrams & Fixed parameters \\
\hline$X_{1}$ & 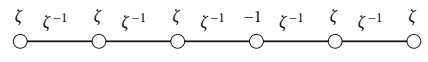 & \\
\hline$X_{2}$ & $\zeta \zeta^{-1} \quad \zeta \zeta^{-1} \quad \zeta^{-1} \overbrace{\zeta}^{\zeta} \overbrace{}^{-1} \zeta^{-1} \zeta$ & \\
\hline$X_{3}$ & $\overbrace{}^{\zeta} \zeta^{-1} \overbrace{}^{\zeta} \zeta^{\zeta^{-1}}{ }^{\zeta}$ & \\
\hline$X_{4}$ & 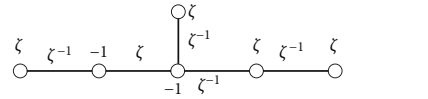 & $\zeta \in G^{\prime}{ }_{3}$ \\
\hline$X_{5}$ & 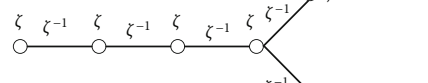 & \\
\hline$X_{6}$ & 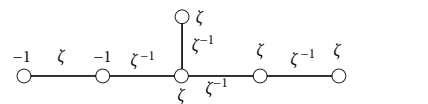 & \\
\hline$X_{7}$ & 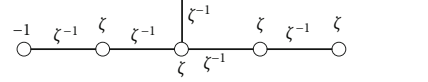 & \\
\hline
\end{tabular}

we collect all objects with generalized Cartan matrixes of type $A_{6}$ in step 4 of the algorithm

3.3. The Algorithms. The fundamental function in Algorithm 28 is the function "Sort":

By Algorithm 30, every root system could be sorted under the same given partial order "cmpvect." Then, comparing and determining whether two root systems are equal are possible.

As a result, we could append new root systems to a container with an order, which is essential in the following function. 
Sort the input root system with the given partial order.

Input: Every rank 6 finite root system in Appendix 5 and function "cmpvect".

Output: An ordered root system, where the order is given by function "cmpvect".

1. The function "cmpvect": given two different roots $\beta_{\mathrm{i}}, \beta_{\mathrm{j}}$ in $r s$, the order of the two roots depends on the natural order of the two integers in the first place $k$ where two roots differ (counting from the beginning of the roots): $\beta_{i}<\beta_{j}$ if and only if $\beta_{i}[k]<\beta_{j}[k]$. Return True, if $\beta_{i}<\beta_{j}$. Else, return False.

2. For roots in $r s$, sort them with the partial order given by "cmpvect" in Step 1. Update $r s$ and return.

Algorithm 30: Sort ( $r s$, cmpvect).

Reflect a root system.

Input: A root system and a direction.

Output: Reflected root systems.

1. Input a root system $r$ in Appendix 5 and direction $i$, where $i \in\{1, \cdots, 6\}$.

2. Calculate the matrix of $i$-th reflection with respect to given standard basis $\alpha_{k}, k \in\{1, \cdots 6\}$, and denote it as $\mathbf{s}_{\mathbf{i}}$.

3 . For every positive root $\alpha$ in the given root system $r s$, calculate $\mathbf{s}_{i} \cdot \alpha$.

4. Construct a set $N$ consisting of $\alpha_{i}$ and $\mathbf{s}_{i} \cdot \alpha$, for all $\alpha$ in the given root system $r s$.

5. Return $N$.

Algorithm 31: Reflection( $r s, i)$.

Remark 32. The reflection in step 2 of Algorithm 31 is given in Equation (13).

Remark 34. The entries of the generalized Cartan matrix of the given root system are defined in Lemma 13.

Finally, we still need a function to check which of the root system is indeed type of $A_{6}$.

\section{Classification Theorem of Finite Cartan Graphs}

Let $M:=\left(\mathbb{k} x_{1}, \cdots, \mathbb{k} x_{6}\right)$ be a tuple of one-dimensional YetterDrinfel'd modules over $\mathbb{k} \otimes G$. Assume that $M$ admits all reflections and $\mathscr{C}(M)=\mathscr{C}\left(I, \mathcal{X}, r,\left(A^{X}\right)_{X \in X}\right)$ be the attached indecomposable semi-Cartan graph to $M$. Assume that $\Delta^{[M]}$ is the set of roots of $\mathscr{B}(M)$. If $\Delta^{[M]}$ is finite, then $\mathscr{C}(M)$ is a finite Cartan graph by Theorem 27.

Theorem 36. Assume $\Delta^{[M]}$ is finite, then there exists a point $X \in \mathscr{X}$ satisfying that the set $\Delta_{+}^{X r e}$ appears in [25] (Corollary 3.13) or in one of the sets listed in Appendix.

Proof. If $\mathscr{C}(M)$ is equivalent to a Cartan graph in [15] (Corollary 3.13$)$, then the claim is true. Otherwise, $\mathscr{C}(M)$ has a unique finite root system by Theorem 27 , say $\mathscr{R}(M)$. Assume that $\mathscr{R}=\mathscr{R}\left(\mathscr{C},\left(\Delta^{\mathrm{X} \text { re }}\right)_{X \in \mathscr{X}}\right)$ is the unique root system, where $\Delta^{X}$ re is the real roots of $X$. Moreover, the root system $\mathscr{R}(M$ ) is irreducible by Proposition 15 . For any $X \in \mathscr{X}$, let $\Delta_{+}^{X \text { re }}$ be the positive roots of $X$. By [25] (Theorem 40), there exists a point $X \in \mathscr{X}$ satisfying that the set $\Delta_{+}^{X}$ re is in the list of Appendix or in [25] (Corollary 3.13) up to a permutation of $I$. There are precisely 4 such possible sets of real roots for the rank 6 case.
The aim of this section is to classify all the finite Cartan graphs $\mathscr{C}(M)$. The output of Algorithm 28 is crucial for the classification of Theorem 39.

In general, the points of a finite Cartan graph $\mathscr{C}$ could have many different neighborhoods. Depending on the output, we construct the following "good $A_{6}$ neighborhoods" in order to cover all the finite connected indecomposable Cartan graphs in such way that at least one point of $\mathscr{C}$ has one of the good neighborhoods.

Definition 37. We say that $X$ has a good $\boldsymbol{A}_{6}$ neighborhood if there exists a permutation of $I$ and an integer $a \in \mathbb{N}$ such that

$$
\begin{aligned}
A^{X}=A^{r_{1}(X)} & =A_{6}, A^{X}=A^{r_{2}(X)}=A_{6}, A^{X}=A^{r_{3}(X)}=A_{6}, \\
A^{r_{4}(X)} & =\left(\begin{array}{cccccc}
2 & -1 & 0 & 0 & 0 & 0 \\
-1 & 2 & -1 & 0 & 0 & 0 \\
0 & -1 & 2 & -1 & -1 & 0 \\
0 & 0 & -1 & 2 & -1 & 0 \\
0 & 0 & -1 & -1 & 2 & -1 \\
0 & 0 & 0 & 0 & -1 & 2
\end{array}\right), A^{r_{5}(X)} \\
& =\left(\begin{array}{cccccc}
2 & -1 & 0 & 0 & 0 & 0 \\
-1 & 2 & -1 & 0 & 0 & 0 \\
0 & -1 & 2 & -1 & 0 & 0 \\
0 & 0 & -1 & 2 & -1 & a \\
0 & 0 & 0 & -1 & 2 & -1 \\
0 & 0 & 0 & a & -1 & 2
\end{array}\right),
\end{aligned}
$$

and $A^{X}=A^{r_{6}(X)}=A_{6}$, where $a$ satisfies one of the following. 
Calculate the generalized Cartan matrix of a root system.

Input: A root system.

Output: The generalized Cartan matrix of the root system.

1. Input a root system $r$ in Appendix 5.

2. Create a matrix $c m$ with entries $a_{i j}$, where $a_{i i}=2, a_{i j}=-\max \left\{k \mid k \alpha_{\mathbf{i}}+\alpha_{\mathbf{j}} \in r s\right\}$, for $i, j \in\{1, \cdots, 6\}$ and $i \neq j$.

3. Return $\mathrm{cm}$.

Algorithm 33: $\mathbf{c m}(r s)$.

Check whether a matrix is type of $A_{6}$.

Input: The generalized Cartan matrix of a root system.

Output: True or False.

1. Construct a matrix $A_{6}=\left(\begin{array}{cccccc}2 & -1 & 0 & 0 & 0 & 0 \\ -1 & 2 & -1 & 0 & 0 & 0 \\ 0 & -1 & 2 & -1 & 0 & 0 \\ 0 & 0 & -1 & 2 & -1 & 0 \\ 0 & 0 & 0 & -1 & 2 & -1 \\ 0 & 0 & 0 & 0 & -1 & 2\end{array}\right)$.

2. If there is a permutation of $\{1, \cdots, 6\}$ satisfying the input matrix $\mathrm{cm}$ is of type $A_{6}$, then return True. Otherwise, return False.

Algorithm 35: IsTypeA6 $(\mathrm{cm})$.

(1) $a=0, a_{36}^{r_{5} r_{4}(X)}=0, a_{32}^{r_{5} r_{4}(X)}=-1, a_{25}^{r_{3} r_{4}(X)}=0, a_{43}^{r_{5} r_{6}(X)}=$ $-1$

(2) $a=-1, a_{36}^{r_{5} r_{4}(X)}=0, a_{25}^{r_{3} r_{4}(X)}=0$

Remark 38. The conditions listed in Definition 37 are possibly not enough to do the classification. In the classification of finite dimensional Nichols algebras of diagonal type over fields of positive characteristic, we need to add more restrictions about the points of the Cartan graph $\mathscr{C}(M)$. For more explanation about how it works, we can check a demonstration in Section 5.

Theorem 39. Assume there exists a point $X \in \mathscr{X}$ satisfying that the set $\Delta_{+}^{X \text { re }}$ appears in Appendix 5 (except $E_{6}$ type), then up to equivalence, there exists a point $Y \in \mathcal{X}$ such that $Y$ has one of the good $A_{6}$ neighborhoods.

Proof. If $\Delta^{[M]}$ appears in Appendix A (except $E_{6}$ type), then there are precisely 3 possible sets of real roots for the rank 6 case. We analyze each set of the real roots in the list. Since the reflection $s_{i}^{X}$ maps $\Delta_{+}^{X \text { re }} \backslash\left\{\alpha_{i}\right\}$ bijectively to $\Delta_{+}^{r_{i}(X) \text { re }} \backslash$ $\left\{\alpha_{i}\right\}$ for any $i \in I$, the Cartan matrices of all neighbors of $X$ can be obtained from $\Delta_{+}^{X \text { re }}$ by Lemma 13 . If there exists a point $Y$ of the Cartan graph $\mathscr{C}(M)$ which has a good $A_{6}$ neighborhood, then the claim is true. Otherwise, repeat the previous step to the neighbors of $X$. Since $\mathscr{X}$ is finite, this algorithm terminates. The elementary calculations are done by GAP Algorithm 1.

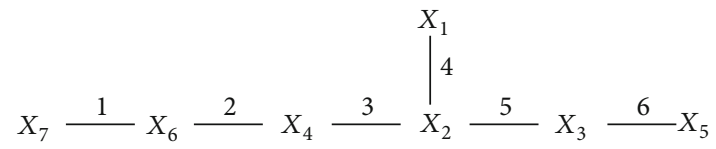

Figure 1: Exchange graph of $\mathscr{C}(M)$.

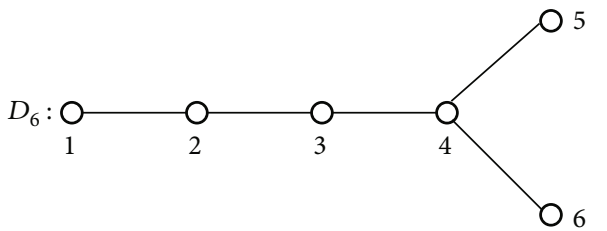

Figure 2: Generalized Dynkin diagram of type $D_{6}$.

\section{Application}

In this section, we present a case of finite dimensional rank 6 Nichols algebras of diagonal type over fields of positive characteristics. We illustrate them with the corresponding generalized Dynkin diagrams and give the exchange graph of the Cartan graph attached to the Nichols algebras.

Theorem 40. Let $\mathbb{k}$ be a field of characteristic $p>0$. Let $I=$ $\{1,2,3,4,5,6\}$. Let $(V, c)$ be a braided vector space of diagonal type over $\mathbb{k}$ with basis $\left\{x_{k} \mid k \in I\right\}$ satisfying

$$
c\left(x_{i} \otimes x_{j}\right)=q_{i j} x_{j} \otimes x_{i} \text { for some } q_{i j} \in \mathbb{k}^{*}
$$


Assume that $\left(q_{i j}\right)_{i, j \in I}$ is an indecomposable braiding matrix. Let $M:=\left(\mathbb{k} x_{i}\right)_{i \in I}$. Assume that the Nichols algebra $\mathscr{B}(V)$ generated by $(V, c)$ has a finite set of roots $\Delta^{[M]}$ listed in Appendix 5 (except $E_{6}$ type). Then the generalized Dynkin diagrams $\mathscr{D}$ of $V$ partially appear in Table 1 . In this case, the row of Table 1 containing $\mathscr{D}$ consists precisely of the generalized Dynkin diagrams of all the points of $\mathscr{C}(M)$. Figure 1 is the exchange graph of the corresponding Cartan graph $\mathscr{C}(M)$.

Remark 41. In the part of the proof, we give the following statement to avoid confusion.

(1) Label the vertices of the generalized Dynkin diagrams from left to right and then top to bottom by $1, \cdots, 6$ (for example: label $D_{6}$ as in Figure 2)

(2) For a generalized Dynkin diagram $\mathscr{D}$ and $i, j, k, l, m$ , $n \in I$, write $\tau_{i j k l m n} \mathscr{D}$ for the graph $\mathscr{D}$ where the vertices of $\mathscr{D}$ change to $i, j, k, l, m, n$, respectively

Proof. Assume that $\mathscr{B}(V)$ has a finite set of roots $\Delta^{[M]}$. Let $X=[M]_{6}^{s}, \quad I=\{1,2,3,4,5,6\}$, and $A^{X}:=\left(a_{i j}\right)_{i, j \in I}$ be the Cartan matrix of $X$. Let $\left(q_{i, j}\right)_{i, j \in I}$ be the braiding matrix of $X$ and $\left(q_{i, j}^{r_{i}(X)}\right)_{i, j \in I}$ be the braiding matrix of $r_{i}(X)$. To simplify the labels, we write $q_{i j}{ }^{\prime}:=q_{i j} q_{j i}$ for $1 \leq i, j \leq 6$. Since $\mathscr{B}(V)$ has a finite set of roots $\Delta^{[M]}$, we obtain that $\mathscr{C}(M)$ is a finite Cartan graph by Theorem 27; we are free to assume that there exists a point $X$ such that $A^{X}$ has a good $A_{6}$ neighborhood by Theorem 39.

Consider the case that $q_{44}=-1$ and $q_{i i} q_{i, i+1}{ }^{\prime}-1=q_{j j}$ $q_{j-1, j}{ }^{\prime}-1=0$ for all $i \in\{1,2,3,5\}$ and $j \in\{2,3,5,6\}$. Let $a$ $:=a_{46}^{r_{5}(X)}$. From Lemma 24, we get $A^{r_{5}(X)}=A_{6}$ and then $a=$ 0 . Let $q:=q_{23}^{\prime}$ and $r:=q_{34}^{\prime}$. Then the condition where $q=r$ $=-1$ does not hold.

Since $X$ has a good $A_{6}$ neighborhood, we obtain $q r \neq 1$. Then, we get 4 th direction reflection
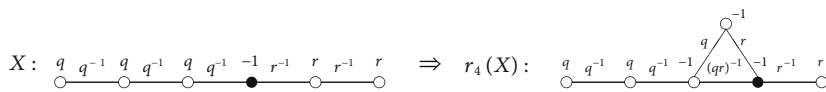

(32)

According to $a_{36}^{r_{5} r_{4}(X)}=0$ by Definition 37 (6), then $q r^{2}=1$ . Hence, we get that

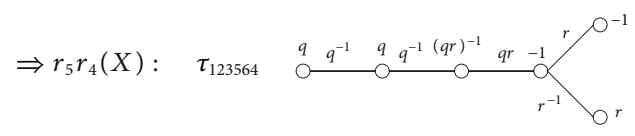

The condition $a_{32}^{r_{5} r_{4}(X)}=-1$ implies that $r q=-1$ or $q^{2} r=1$ by Lemma 24 . If $r q=-1$ then $r=-1, q=1$, which is a contradiction. If $q^{2} r=1$, then $q=r$ and $q^{3}=1$. Hence,

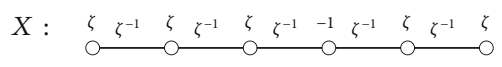

where $\zeta \in G^{\prime}{ }_{3}$. We denote it as $X_{1}$ in Table 1 . Doing all the reflections of $X_{1}$, we obtain the generalized Dynkin diagrams $X_{2}, X_{3}, X_{4}, X_{5}, X_{6}$, and $X_{7}$ in Table 1 . And the corresponding exchange graph of $\mathscr{C}(M)$ is Figure 1 .

\section{Appendix}

\section{The Input Irreducible Root Systems of Rank 6}

Nr. 1 with 36 positive roots (type $E_{6}$ ):

$1,2,3,4,5,6,12,13,14,25,36,123,124,125,134,136$, $1234,1235,1236,1245,1346,1^{2} 234,12345,12346,12356$, $1^{2} 2345,1^{2} 2346,123456,1^{2} 2^{2} 345,1^{2} 23^{2} 46,1^{2} 23456,1^{2} 2^{2}$ $3456,1^{2} 23^{2} 456,1^{2} 2^{2} 3^{2} 456,1^{3} 2^{2} 3^{2} 456,1^{3} 2^{2} 3^{2} 4^{2} 56$

Nr. 2 with 46 positive roots:

$1,2,3,4,5,6,12,13,14,23,25,46,123,124,125,134,146$, $235,1234,1235,1245,1246,1346,1^{2} 234,12^{2} 35,12345$, $12346,12456,1^{2} 2345,1^{2} 2346,12^{2} 345,123456,1^{2} 2^{2} 345,1^{2}$ $234^{2} 6,1^{2} 23456,12^{2} 3456,1^{2} 2^{2} 3^{2} 45,1^{2} 2^{2} 3456,1^{2} 234^{2} 56,1^{2}$ $2^{2} 3^{2} 456,1^{2} 2^{2} 34^{2} 56,1^{3} 2^{2} 34^{2} 56,1^{2} 2^{2} 3^{2} 4^{2} 56,1^{3} 2^{2} 3^{2} 4^{2} 56,1^{3}$ $2^{3} 3^{2} 4^{2} 56,1^{3} 2^{3} 3^{2} 4^{2} 5^{2} 6$

Nr. 3 with 63 positive roots:

$1,2,3,4,5,6,12,13,14,23,35,56,123,124,134,135,235$, $356,1^{2} 24,1234,1235,1345,1356,2356,1^{2} 234,123^{2} 5,12345$, $12356,13456,1^{2} 2^{2} 34,1^{2} 2345,123^{2} 45,123^{2} 56,123456,1^{2} 2^{2}$ $345,1^{2} 23^{2} 45,1^{2} 23456,123^{2} 456,123^{2} 5^{2} 6,1^{2} 2^{2} 3^{2} 45,1^{2} 2^{2}$ $3456,1^{2} 23^{2} 456,123^{2} 45^{2} 6,1^{3} 2^{2} 3^{2} 45,1^{2} 2^{2} 3^{2} 456,1^{2} 23^{2} 45^{2} 6$, $1^{3} 2^{2} 3^{2} 4^{2} 5,1^{3} 2^{2} 3^{2} 456,1^{2} 2^{2} 3^{2} 45^{2} 6,1^{2} 23^{3} 45^{2} 6,1^{3} 2^{2} 3^{2} 4^{2} 56$, $1^{3} 2^{2} 3^{2} 45^{2} 6,1^{2} 2^{2} 3^{3} 45^{2} 6,1^{3} 2^{2} 3^{3} 45^{2} 6,1^{3} 2^{2} 3^{2} 4^{2} 5^{2} 6,1^{3} 2^{3} 3^{3} 45^{2}$ $6,1^{3} 2^{2} 3^{3} 4^{2} 5^{2} 6,1^{4} 2^{2} 3^{3} 4^{2} 5^{2} 6,1^{3} 2^{3} 3^{3} 4^{2} 5^{2} 6,1^{4} 2^{3} 3^{3} 4^{2} 5^{2} 6,1^{4} 2^{3}$ $3^{4} 4^{2} 5^{2} 6,1^{4} 2^{3} 3^{4} 4^{2} 5^{3} 6,1^{4} 2^{3} 3^{4} 4^{2} 5^{3} 6^{2}$

Nr. 4 with 68 positive roots:

$1,2,3,4,5,6,12,13,14,35,56,1^{2} 2,123,124,134,135$, $356,1^{2} 23,1^{2} 24,1234,1235,1345,1356,1^{2} 234,1^{2} 235$, $12345,12356,13456,1^{3} 234,1^{2} 23^{2} 5,1^{2} 2345,1^{2} 2356,123456$ $, 1^{3} 2^{2} 34,1^{3} 2345,1^{2} 23^{2} 45,1^{2} 23^{2} 56,1^{2} 23456,1^{3} 2^{2} 345,1^{3} 23^{2}$ $45,1^{3} 23456,1^{2} 23^{2} 456,1^{2} 23^{2} 5^{2} 6,1^{3} 2^{2} 3^{2} 45,1^{3} 2^{2} 3456,1^{3} 23^{2}$ $456,1^{2} 23^{2} 45^{2} 6,1^{4} 2^{2} 3^{2} 45,1^{3} 2^{2} 3^{2} 456,1^{3} 23^{2} 45^{2} 6,1^{4} 2^{2} 3^{2} 4^{2} 5$, $1^{4} 2^{2} 3^{2} 456,1^{3} 2^{2} 3^{2} 45^{2} 6,1^{3} 23^{3} 45^{2} 6,1^{4} 2^{2} 3^{2} 4^{2} 56,1^{4} 2^{2} 3^{2} 45^{2} 6$, $1^{3} 2^{2} 3^{3} 45^{2} 6,1^{4} 2^{2} 3^{3} 45^{2} 6,1^{4} 2^{2} 3^{2} 4^{2} 5^{2} 6,1^{5} 2^{2} 3^{3} 45^{2} 6,1^{4} 2^{2} 3^{3} 4^{2}$ $5^{2} 6,1^{5} 2^{3} 3^{3} 45^{2} 6,1^{5} 2^{2} 3^{3} 4^{2} 5^{2} 6,1^{5} 2^{3} 3^{3} 4^{2} 5^{2} 6,1^{6} 2^{3} 3^{3} 4^{2} 5^{2} 6,1^{6}$ $2^{3} 3^{4} 4^{2} 5^{2} 6,1^{6} 2^{3} 3^{4} 4^{2} 5^{3} 6,1^{6} 2^{3} 3^{4} 4^{2} 5^{3} 6^{2}$

\section{Data Availability}

The data used to support the findings of this study is available inside the paper.

\section{Disclosure}

Since we are dealing with the finite dimensional Nichols algebras and related structures, the backgrounds in Introduction and the terminology in Section 2 are similar to that of the corresponding author's paper [37] (Introduction and Section 1) submitted as preprint in arXiv.org with the link http://arxiv.org/abs/1911.03555 and published in the Journal of Algebra [37]. 


\section{Conflicts of Interest}

The authors declare that they have no conflicts of interest.

\section{References}

[1] N. Andruskiewitsch, "On finite-dimensional Hopf algebras," International Congress of Mathematicians, vol. 2, pp. 117141, 2014.

[2] A. M. Semikhatov, "Virasoro central charges for Nichols algebras," in Conformal Field Theories and Tensor Categories. Mathematical Lectures from Peking University, C. Bai, J. Fuchs, Y. Z. Huang, L. Kong, I. Runkel, and C. Schweigert, Eds., pp. 67-92, Springer, Berlin, Heidelberg, 2014.

[3] A. M. Semikhatov and I. Y. Tipunin, "The Nichols algebra of screenings," Communications in Contemporary Mathematics, vol. 14, no. 4, article 1250029, 2012.

[4] A. M. Semikhatov and I. Y. Tipunin, "Logarithmic $s l_{2}$ CFT models from Nichols algebras: I," Journal of Physics A: Mathematical and Theoretical, vol. 46, no. 49, article 494011, 2013.

[5] N. Andruskiewitsch, "About finite dimensional Hopf algebras," Contemporary Mathematics, vol. 294, pp. 1-57, 2002.

[6] N. Andruskiewitsch, I. Heckenberger, and H.-J. Schneider, "Braided Hopf algebras over non abelian finite groups," Boletin de la Academia Nacional de Ciencias en Córdoba, vol. 63, pp. 45-78, 1998.

[7] N. Andruskiewitsch and H.-J. Schneider, "Pointed Hopf algebras," Mathematical Sciences Research Institute Publications, vol. 43, p. 10, 2001.

[8] P. Schauenburg, "A characterization of the Borel-like subalgebras of quantum enveloping algebras," Communications in Algebra, vol. 24, no. 9, pp. 2811-2823, 1996.

[9] N. Andruskiewitsch and H.-J. Schneider, "Lifting of quantum linear spaces and pointed Hopf algebras of order $p^{3}$," Journal of Algebra, vol. 209, no. 2, pp. 658-691, 1998.

[10] W. D. Nichols, "Bialgebras of type one," Communications in Algebra, vol. 6, no. 15, pp. 1521-1552, 1978.

[11] G. Lusztig, "Noncommutative differentials and Yang-Mills on permutation groups sn," in Lecture Notes in Pure and Applied Mathematics, vol. 239, CRC Press, 2001.

[12] G. Lusztig, Introduction to Quantum Groups, Springer Science \& Business Media, 2010

[13] M. Rosso, "Quantum groups and quantum shuffles," Inventiones Mathematicae, vol. 133, no. 2, pp. 399-416, 1998.

[14] S. L. Woronowicz, "Compact matrix pseudogroups," Communications in Mathematical Physics, vol. 111, no. 4, pp. 613-665, 1987.

[15] S. L. Woronowicz, "Differential calculus on compact matrix pseudogroups (quantum groups)," Communications in Mathematical Physics, vol. 122, no. 1, pp. 125-170, 1989.

[16] Y. Bahturin, A. V. Mikhalev, V. M. Petrogradsky, and M. V. Zaicev, Infinite Dimensional Lie Superalgebras, Walter de Gruyter, 1992.

[17] V. Kharchenko, "A quantum analog of the Poincare-BirkhoffWitt theorem," Algebra and Logic, vol. 38, no. 4, pp. 259-276, 1999.

[18] I. Heckenberger, "The Weyl groupoid of a Nichols algebra of diagonal type," Inventiones Mathematicae, vol. 164, no. 1, pp. 175-188, 2006.
[19] M. Cuntz and I. Heckenberger, "Weyl groupoids with at most three objects," Journal of Pure and Applied Algebra, vol. 213, no. 6, pp. 1112-1128, 2009.

[20] I. Heckenberger and H. Yamane, "A generalization of Coxeter groups, root systems, and Matsumoto's theorem," Mathematische Zeitschrift, vol. 259, no. 2, pp. 255-276, 2008.

[21] I. Heckenberger and H.-J. Schneider, "The Nichols algebra of a semisimple Yetter-Drinfeld module," American journal of mathematics, vol. 132, no. 6, pp. 1493-1547, 2010.

[22] I. Heckenberger and H.-J. Schneider, "Root systems and Weyl groupoids for Nichols algebras," Proceedings of the London Mathematical Society, vol. 101, no. 3, pp. 623-654, 2010.

[23] I. Heckenberger and H.-J. Schneider, "Right coideal subalgebras of Nichols algebras and the duflo order on the Weyl groupoid," Israel Journal of Mathematics, vol. 197, no. 1, pp. 139-187, 2013.

[24] M. Cuntz and I. Heckenberger, "Finite Weyl groupoids of rank three," Transactions of the American Mathematical Society, vol. 364, no. 3, pp. 1369-1393, 2012.

[25] M. Cuntz and I. Heckenberger, "Finite Weyl groupoids," Journal für die reine und angewandte Mathematik (Crelles Journal), vol. 2015, no. 702, pp. 77-108, 2015.

[26] N. Andruskiewitsch and H.-J. Schneider, "Finite quantum groups and Cartan matrices," Advances in Mathematics, vol. 154, no. 1, pp. 1-45, 2000.

[27] I. Heckenberger, "Classification of arithmetic root systems of rank 3," 2005, https://arxiv.org/abs/math/0509145.

[28] I. Heckenberger, "Weyl equivalence for rank 2 Nichols algebras of diagonal type," Annali dell'Università di Ferrara, vol. 51, no. 1, pp. 281-289, 2005.

[29] I. Heckenberger, "Examples of finite-dimensional rank 2 Nichols algebras of diagonal type," Compositio Mathematica, vol. 143, no. 1, pp. 165-190, 2007.

[30] I. Heckenberger, "Classification of arithmetic root systems," Advances in Mathematics, vol. 220, no. 1, pp. 59-124, 2009.

[31] I. Angiono, "On Nichols algebras of diagonal type," Journal fur die Reine und Angewandte Mathematik, vol. 2013, no. 683, pp. 189-251, 2013.

[32] I. Angiono, "A presentation by generators and relations of Nichols algebras of diagonal type and convex orders on root systems," Journal of the European Mathematical Society, vol. 17, no. 10, pp. 2643-2671, 2015.

[33] N. Andruskiewitsch and H.-J. Schneider, "On the classification of finite-dimensional pointed Hopf algebras," Annals of Mathematics, vol. 171, no. 1, pp. 375-417, 2010.

[34] C. Cibils, A. Lauve, and S. Witherspoon, "Hopf quivers and Nichols algebras in positive characteristic," Proceedings of the American Mathematical Society, vol. 137, no. 12, pp. 40294041, 2009.

[35] I. Heckenberger and J. Wang, "Rank 2 Nichols algebras of diagonal type over fields of positive characteristic," SIGMA. Symmetry, Integrability and Geometry: Methods and Applications, vol. 11, p. 11, 2015.

[36] J. Wang, "Rank three Nichols algebras of diagonal type over arbitrary fields," Israel Journal of Mathematics, vol. 218, no. 1, pp. 1-26, 2017.

[37] J. Wang, "Rank 4 finite-dimensional Nichols algebras of diagonal type in positive characteristic," Journal of Algebra, vol. 559, pp. 547-579, 2020. 
[38] M. Takeuchi, "Survey of braided hopf algebras," Contemporary Mathematics, vol. 267, pp. 301-324, 2000.

[39] M. Cuntz and I. Heckenberger, "Weyl groupoids of rank two and continued fractions," Algebra Number Theory, vol. 3, no. 3, pp. 317-340, 2009.

[40] I. Heckenberger, "Rank 2 Nichols algebras with finite arithmetic root system," Algebras and Representation theory, vol. 11, no. 2, pp. 115-132, 2008. 